\title{
Recurso polínico y ciclo estacional de Thygater aethiops (Hymenoptera: Apidae) en un ambiente urbano (Bogotá-Colombia)
}

\author{
Mario Simón Pinilla-Gallego ${ }^{1}$, Valentina Nieto Fernández ${ }^{2} \&$ Guiomar Nates-Parra ${ }^{3}$ \\ Laboratorio de Investigación en Abejas-LABUN, Departamento de Biología, Facultad de Ciencias, Universidad \\ Nacional de Colombia, Av. Carrera 30 \# 45-03, Ciudad Universitaria, Edificio 421, Oficina 128, Bogotá, D.C., \\ Colombia; simonpinilla@yahoo.com, vnietof@unal.edu.co,mgnatesp@unal.edu.co
}

Recibido 24-VIII-2015. Corregido 08-III-2016. ～Aceptado 05-IV-2016.

\begin{abstract}
Pollen resource and seasonal cycle of Thygater aethiops (Hymenoptera: Apidae) in an urban environment (Bogotá-Colombia). Thygater aethiops is a native bee that can be found in parks and gardens in diverse urban areas such as those in the city of Bogotá (Colombia). However, little is known about its biology as well as ecological adaptations to urban areas. This study aimed to describe the seasonal cycle and daily foraging activities of $T$. aethiops, as well as identify the pollen resources used by this bee over a year in a population nesting in an aggregation in the "Parque Nacional Enrique Olaya Herrera" in Bogotá. Changes in the nest activity were monitored weekly by counting the number of active nests in the aggregation between December/2012 and February/2014. To determine the daily foraging activity, the numbers of bees entering their nests over a period of 10 minutes every hour between 8:00 and 14:00 h were recorded. Females with pollen loads entering to their nest were captured weekly, between September/2012 and August/2013, and their pollen loads analyzed. Three nesting peaks occurred after the precipitation peaks, but the number of active nests was not correlated with precipitation. The nesting activities stopped in a large number of the active nests $(20-50 \%$ of nests) after an anthropic disturbance was registered in the nesting area. Bees forage for nectar and pollen between 8:00 and 14:00 h, with a peak at 10:00 h. Daily foraging activity changed during the study period due to anthropic disturbance. There was not a significant relationship between air temperature and the number of females entering their nests. Foraging activities did not change between the dry and rainy seasons. A total of 26 pollen types were found in 169 pollen loads. Ulex europaeus (Fabaceae) and Solanum laxum (Solanaceae) were the most abundant plants represented on the pollen load across the study period. According to these results, $T$. aethiops would be considered a mesolectic species. The ability of $T$. aethiops to use different pollen resources both native and exotic, as well as to presumably recover its population after disturbances, are characteristics that may have allowed this bee to adapt to urban environments. Knowledge on the floral resources as well as other biological features of this bee species is important to promote its conservation in urban areas. Rev. Biol. Trop. 64 (3): 1247-1257. Epub 2016 September 01.
\end{abstract}

Key words: urban bees, conservation, Eucerini, Solanum laxum, Ulex europaeus.

Las abejas (Hymenoptera: Apoidea) son consideradas especies clave en muchos ecosistemas naturales así como en agroecosistemas, por su importante papel en la polinización (Kratochwil, 2003; Vaissiere, Freitas, \& Gemmill-Herren, 2011). Los Andes constituyen una región con alta diversidad de abejas, y para Colombia se han registrado alrededor de 68 especies en dicha zona (González, VelezRuiz, \& Engel, 2014). Aunque la mayoría de los estudios de vida silvestre se llevan a cabo en zonas relativamente conservadas, también se ha observado la importancia de estudiar las especies que han logrado adaptarse a zonas altamente intervenidas. La ecología urbana es un campo de estudio en crecimiento, y son escasos los reportes ecológicos de abejas urbanas (Hernández, Frankie, \& Thorp, 2009). Los hábitats urbanos se caracterizan por una alta fragmentación y frecuencia de disturbios, 
así como vegetación fuertemente alterada o exótica (Mcintyre \& Hostetler, 2001; Pellkofer, 2011). Aun así, algunas especies de abejas han logrado adaptarse a estos ambientes, siendo las especies generalistas y aquellas que nidifican en cavidades preexistentes, las que más suelen encontrarse en zonas urbanas (Mcintyre \& Hostetler, 2001; Hernández et al., 2009; Dalmazzo, 2010).

En Bogotá (Colombia) y sus alrededores Nates-Parra, Parra, Rodríguez, Baquero and Vélez (2006) registraron 40 especies de abejas. Una de estas especies es Thygater aethiops Smith, 1854 (Hymenoptera: Apidae: Eucerini); es de tamaño medio $(13-15 \mathrm{~mm})$, solitaria, nidifica en el suelo y es capaz de realizar polinización por zumbido (González, Ospina, \& Bennet, 2005; Nates-Parra et al., 2006) lo que la convierte en un potencial polinizador de plantas como el tomate (Solanum lycopersicum). Los nidos de T. aethiops se pueden encontrar de forma solitaria o en agregados, las entradas de los nidos son circulares y cuando el nido está activo se puede observar acumulaciones de tierra cerca de la entrada (Quijano, Parra, \& Nates-Parra, 2002; Nates-Parra et al., 2006).

En Bogotá, T. aethiops se puede encontrar en zonas rurales, así como en amplias zonas verdes dentro de la ciudad, como el Jardín Botánico de Bogotá y el Parque Nacional Enrique Olaya Herrera, en donde estas abejas nidifican y aprovechan la vegetación existente como fuente de alimento y como lugar de descanso y apareamiento (Nates-Parra et al., 2006). Sin embargo, es poco lo que se conoce sobre la biología de esta especie y como ha podido sobrevivir en medio de una zona fuertemente alterada. Debido a esto, el objetivo de este estudio fue describir posibles ciclos estacionales y horarios de actividad de T. aethiops, así como identificar el recurso polínico usado a lo largo de un año, en los nidos presentes en el Parque Nacional Enrique Olaya Herrera de Bogotá (Colombia).

\section{MATERIALES Y MÉTODOS}

Área de estudio: Bogotá (Colombia) tiene un área total de $1605 \mathrm{~km}^{2}$ y un área urbana de $307 \mathrm{~km}^{2}$; se estima que para el 2014 la población era de 7776845 habitantes (DANE, 2015). Históricamente, el régimen de precipitación es bimodal, con mayor precipitación entre los meses de marzo a mayo y septiembre a noviembre (IDEAM, 2014). El estudio se llevó a cabo en el Parque Nacional Enrique Olaya Herrera (PNEOH), el cual limita al oriente con los cerros orientales de Bogotá, mientras que por el norte, sur, y oeste, se encuentra rodeado por la ciudad. El PNEOH cuenta con un área de 283 ha, de las cuales 141 son reserva forestal; en el área restante se disponen zonas verdes, jardines y escenarios deportivos y culturales (IDRD, 2014).

Las observaciones se hicieron en un agregado de nidos localizado en el sector histórico del parque $\left(4^{\circ} 37^{\prime} 27^{\prime \prime} \mathrm{N}-7^{\circ} 03^{\prime} 51^{\prime \prime} \mathrm{W}, 2605\right.$ $\mathrm{msnm}$ ), en una rotonda peatonal que presenta un hundimiento en su base aprovechado por T. aethiops para construir sus nidos (Fig. 1A). Debido a la estructura de la rotonda, el agregado de nidos se dispone de forma lineal con aproximadamente $30 \mathrm{~m}$ de longitud, rodeado de jardines y zonas arboladas del parque, y con frecuente tránsito de personas.

Estacionalidad: Se realizaron observaciones semanales desde diciembre/2012 hasta febrero/2014 (15 meses) en las que se contó el número de nidos activos en el agregado, tomando como nidos activos aquellos que presentaban acumulación de tierra en la entrada (González \& Ospina, 2008). También se tomó nota de la presencia de machos en flores cercanas al agregado.

Horario de entradas a los nidos: Para determinar el rango general de actividad de entrada a los nidos se efectuaron dos muestreos preliminares entre las 7:00 y las 16:00 h. Posteriormente, se realizaron observaciones semanales desde septiembre/2012 hasta agosto/2013 (12 meses). En cada observación se contó el número de abejas que entraban a sus nidos en un periodo de 10 min cada hora, entre las 8:00 y las 14:00 h, a lo largo de $1 \mathrm{~m}$ lineal en la rotonda donde se encontraba el agregado de 

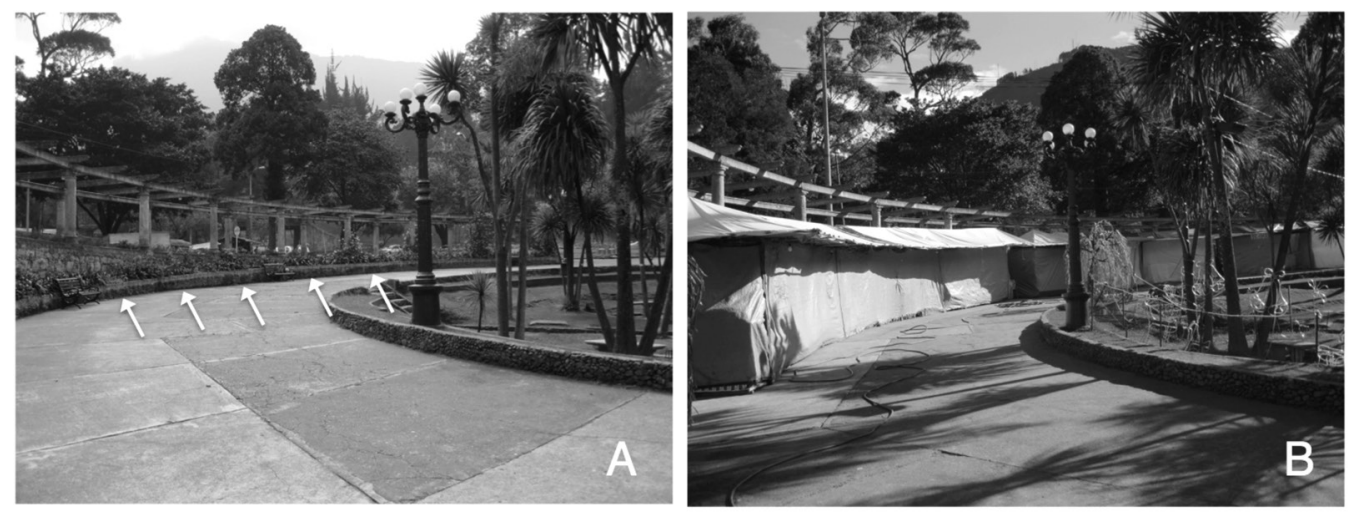

Fig. 1. (A) Rotonda en la que se encuentra el agregado de nidos de T. aethiops. Las flechas indican la ubicación de los nidos en la rotonda. (B) Carpas comerciales instaladas en la rotonda tres veces al año donde se encontraba el agregado de nidos. Fig. 1. (A) Roundabout in which the nest aggregation of $T$. aethiops was located. Arrows indicate the nest location at the roundabout. (B) Commercial tent installed at the roundabout three times a year where the nests aggregation was located.

nidos. Se diferenció entre las abejas que entraban a los nidos con cargas de polen, y aquellas que entraban sin carga de polen.

Identificación del recurso polínico: Se realizaron muestreos semanales desde septiembre/2012 hasta agosto/2013 (12 meses). En cada muestreo se capturó entre cuatro y seis hembras con cargas de polen en las escopas mientras regresaban a los nidos. Las abejas se introdujeron en frascos de plástico y luego fueron puestas en hielo para inmovilizar los individuos, y así retirar la carga de polen, después de lo cual las abejas se liberaron. Las muestras de polen se llevaron al Laboratorio de Melisopalinología de la Universidad Nacional de Colombia sede Bogotá, donde se les aplicó el proceso de acetólisis, siguiendo el protocolo de Fonnegra (1989). El polen acetolizado se montó en láminas portaobjetos y se observó al microscopio óptico. En cada lámina se contó un total de 300 granos de polen (Louveaux, Maurizio, \& Vorwohl, 1978), identificando el origen botánico hasta el nivel taxonómico más bajo posible. Para identificar el polen, se recurrió a la palinoteca del Laboratorio de Melisopalinología, y a muestras de polen de flores recolectadas en el PNEOH durante el periodo de estudio.

Se obtuvieron los datos diarios de precipitación y temperatura del aire de la estación meteorológica del Departamento de Ingeniería Civil y Agrícola de la Universidad Nacional de Colombia, ubicada a $2.3 \mathrm{~km}$ del PNEOH. Se realizó un análisis de correlación de Pearson entre la precipitación semanal y el número de nidos activos en cada semana. Adicionalmente, se realizó una regresión lineal entre la temperatura del aire registrada durante cada hora de muestreos y los datos de entradas a los nidos. Para todos los análisis se utilizó el software R 3.1 (R Core Team, 2014).

Durante el año de muestreo se observaron cambios en el patrón del horario de entradas a los nidos de $T$. aethiops por lo que los promedios del horario de actividad fueron calculados en tres periodos de cuatro meses cada uno de la siguiente manera: Periodo 1, septiembre/2012diciembre/2012; periodo 2, enero/2013abril/2013; periodo 3, mayo/2013-agosto/2013.

Para analizar el horario de entradas en lo nidos en función del periodo climático, se generaron promedios del horario de actividad para la época lluviosa (febrero-mayo 2013) y época seca (junio-agosto 2013). Estos periodos fueron determinados con los datos obtenidos de la estación meteorológica.

Para calcular el aporte total y mensual de cada tipo polínico se utilizó la corrección volumétrica propuesta por Da Silveira (1991). Se usó la prueba de Shapiro-Wilk para determinar 
la distribución normal, y la prueba de Bartlett para evaluar la homogeneidad de varianzas. Los datos corregidos de polen fueron trasformados por raíz cuadrada debido a que no presentaron una distribución normal. Se comparó el aporte de cada tipo polínico durante cada mes de muestreo, así como el aporte total a lo largo de todo el año de estudio. Para esto se aplicó la prueba paramétrica de análisis de varianza (ANOVA) de una vía con un nivel de significancia de $P \leq 0.05$. En caso de encontrarse diferencias significativas se utilizó la prueba de Tukey para determinar qué tratamiento ( $=$ tipo polínico) fue diferente $(\mathrm{p} \leq 0.05)$. Cargas en las que más del $90 \%$ del polen correspondía a un solo taxón fueron consideradas monoflorales (Sipes \& Tepedino, 2005).

\section{RESULTADOS}

Estacionalidad: El número de nidos activos mostró tres picos a lo largo del periodo de estudio durante los meses de mayo/2013, octubre/2013 y febrero/2014. Los periodos con menor número de nidos activos fueron enero/2013, agosto/2013 y diciembre/2013 (Fig. 2). No se observó correlación entre el número de nidos activos y la precipitación (Pearson, $\mathrm{df}=44 ; \mathrm{t}=0.861 ; \mathrm{P}>0.05$ ). Los machos se observaron en flores del parque durante todo el periodo de muestreo.
Durante los meses de estudio, en tres ocasiones la administración del parque instaló una serie de carpas comerciales a lo largo de la rotonda en la que se encontraba el agregado de nidos (Fig. 1B). Estas carpas no formaban parte del diseño experimental. La primera instalación ocurrió entre diciembre/2012-enero/2013; después de retiradas, el número de nidos activos se redujo $51.1 \%$. Las carpas fueron instaladas nuevamente en octubre/2013 (durante una semana) y entre diciembre/2013-enero/2014, con reducciones del 43.7 y $20.2 \%$ en el número de nidos activos respectivamente (Fig. 2).

Horario de entradas a los nidos: La actividad de entradas en los nidos inicia a las 8:00 h y finaliza cerca de las 14:00 h; dicha actividad no mostró relación con la temperatura del aire $\left(d f=1\right.$ y $\left.272 ; \mathrm{t}=-0.768 ; \mathrm{p}>0.05 ; \mathrm{R}^{2}=0.00216\right)$. Durante el primer periodo se presentó un pico de actividad a las 10:00 h, que corresponde a un pico en el número de entradas de hembras con polen. El número de entradas sin polen alcanzó su valor máximo a las 11:00 h, después de lo cual la actividad disminuyó (Fig. 3A).

Después de la instalación de las carpas comerciales en diciembre/2012 se observó un cambio en el horario de actividad de las abejas. En el segundo periodo, posterior al disturbio producido por las carpas, se registró un descenso en el número de individuos observados

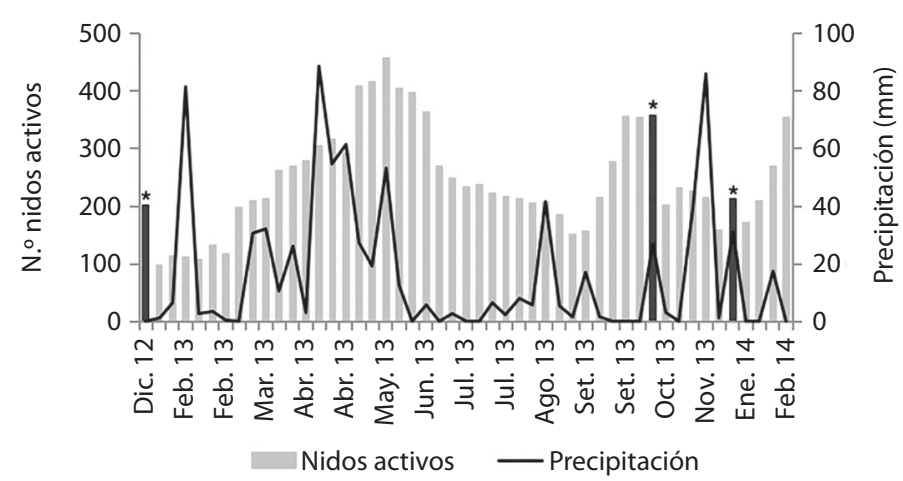

Fig. 2. Precipitación y número de nidos activos total en el agregado de T. aethiops a lo largo de 15 meses. Las barras oscuras y marcadas con un asterisco indican las semanas en que se instalaron carpas comerciales en la rotonda.

Fig. 2. Precipitation and total number of active nests in the aggregation of T. aethiops along 15 months. Dark bars marked with an asterisk indicate the week in which commercial tent were installed in the roundabout. 


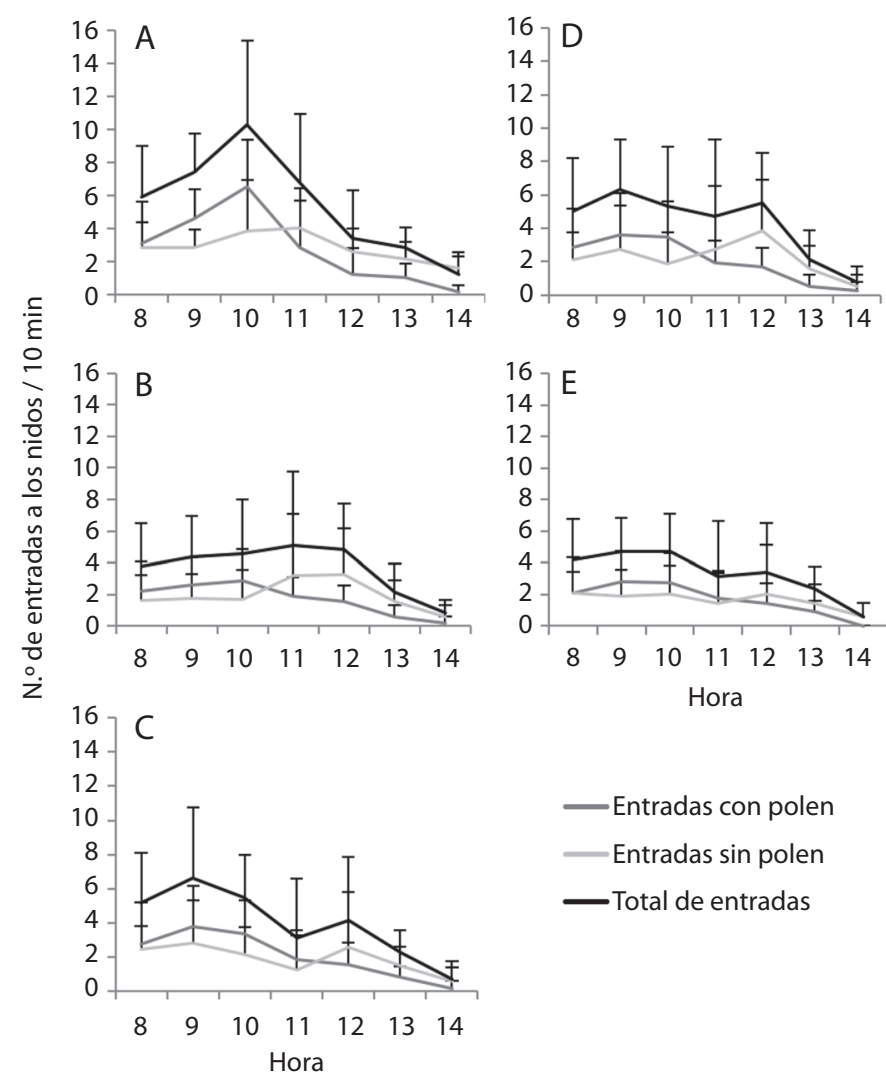

Fig. 3. Número de entradas a los nidos (promedio \pm DE) (A) antes de un disturbio (septiembre, octubre, noviembre y diciembre 2012), (B) en los meses posteriores al disturbio (enero, febrero, marzo y abril 2013), (C) y transcurrido un periodo de recuperación (mayo, junio, julio y agosto 2013), así como durante (D) la temporada de lluvias y (E) temporada seca, en el agregado de nidos de T. aethiops.

Fig. 3. Number of nests entries (average \pm SD) (A) before a disturbance (September, October, November and December of 2012), (B) in the months after disturbance (January, February, March and April of 2013), (C) and after a period of recovery (May, June, July, and August of 2013), as well as during (D) rain season and (E) dry season in the nest aggregation of T. aethiops.

durante los muestreos, además no se observó el pico de actividad de entradas con polen a las 10:00 h, mientras que, aunque menos pronunciado, se mantuvo el pico de actividad de las 11 h (Fig. 3B). Durante el tercer periodo el número de individuos observados aumentó nuevamente. En esta ocasión el pico de actividad total se registró a las 9:00 h, el pico de entradas con polen fue leve y se registró también a las 9:00 $\mathrm{h}$, mientras que el pico de entradas sin polen se registró a las 12:00 h (Fig. 3C).

El horario de entradas a los nidos mostró el mismo patrón en época lluviosa y seca, con mayor recolección de polen entre las 8:00-10:00 $\mathrm{h}$, luego de lo cual aumentó el número de entradas sin polen hasta las 12:00 h. La actividad de entradas a los nidos cesa cerca de las 14:00 h. El número total de individuos observados fue menor en época seca (Fig. 3D y Fig. 3E).

Recurso polínico: Se obtuvo un total de 169 cargas de polen de T. aethiops, de las cuales el $85.2 \%$ fue monofloral y el $14.8 \%$ bifloral. Se encontraron 26 tipos polínicos, siendo 16 de ellos nuevos registros de plantas visitadas por T. aethiops. Ulex europaeus (Fabaceae) y Solanum laxum (Solanaceae) fueron las plantas con mayor aporte total al recurso polínico 
(Tukey, $\mathrm{df}=25 ; \mathrm{F}=52.51 ; \mathrm{P}<0.01)($ Cuadro 1$)$. Ulex europaeus brindó un aporte significativamente mayor durante cinco meses, mientras que $S$. laxum mostró un aporte significativamente mayor durante tres meses. En los meses restantes, dos o más especies presentaron aportes estadísticamente similares (Fig. 4).

\section{DISCUSIÓN}

Aunque algunos autores han estudiado el recurso floral utilizado por $T$. aethiops (Nates-Parra et al., 2006; González \& Ospina, 2008) este es el primer reporte basado en el análisis de cargas de polen. Adicionalmente,

\section{CUADRO 1}

Tipos polínicos más frecuentes encontrados en las cargas de polen de T. aethiops $(\mathrm{n}=169)$ y su aporte al recurso polínico total

TABLE 1

Most frequent pollen types found in T. aethiops pollen loads $(n=169)$ and its contribution to the total pollen resource

\begin{tabular}{llc}
\multicolumn{1}{c}{ Familia } & \multicolumn{1}{c}{ Especie } & Aporte total al recurso polínico $(\%)^{*}$ \\
Solanaceae & Ulex europaeus & $63.7 \mathrm{a}$ \\
Fabaceae & Solanum laxum & $27.8 \mathrm{~b}$ \\
Salicaceae & Abatia parviflora & $4.7 \mathrm{c}$ \\
Melastomataceae & Tibouchina lepidota & $2.5 \mathrm{~cd}$ \\
Lamiaceae & sp. & $0.4 \mathrm{~cd}$ \\
Escalloniaceae & Escallonia sp. & $0.2 \mathrm{~d}$ \\
\hline
\end{tabular}

Tipos polínicos con baja frecuencia de aparición se presentan en el Apéndice 1.

Pollen types with low frequency of occurrence are presented in Appendix 1.

* letras diferentes en la misma columna representan diferencias significativas $(\mathrm{P}<0.05)$ de acuerdo a la prueba de Tukey.
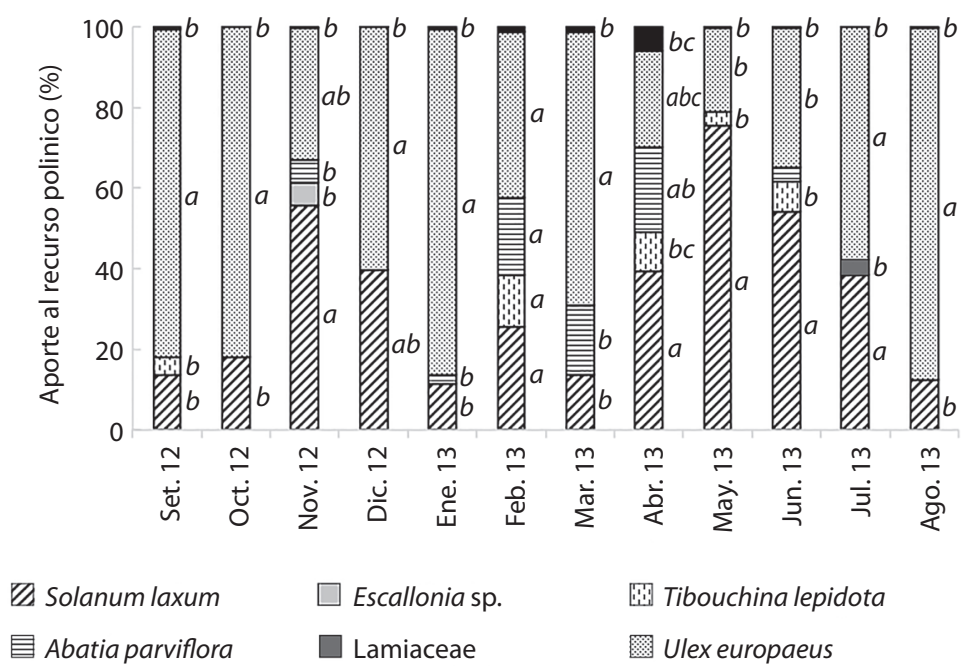

$\square$ Otros

Fig. 4. Aporte mensual de cada especie vegetal al recurso polínico de T. aethiops. Aquellos tipos polínicos con un aporte menor al $2 \%$ mensual fueron agrupados dentro de la categoría "otros". Letras diferente en la misma columna representan diferencias significativas $(\mathrm{P}<0.05)$ de acuerdo a la prueba de Tukey.

Fig. 4. Monthly contribution of each plant species to the pollen resource of $T$. aethiops. Those pollen types with monthly contribution less than $2 \%$ were grouped in the category "otros (=other)". Different letters in the same column represent significant differences $(\mathrm{P}<0.05)$ according to Tukey test. 
se registraron 16 nuevas plantas visitadas por esta especie con lo que actualmente se reporta un total de 76 plantas utilizadas por T. aethiops (Apéndice 1). Debido a esto, T. aethiops ha sido catalogada como una especie generalista, sin embargo, en este estudio observamos que solo seis especies contribuyen regularmente a la dieta de T. aethiops. De acuerdo a la clasificación propuesta por Cane y Sipes (2006) para el nivel de especialización de las abejas, $T$. aethiops sería considerada una especie mesoléctica, ya que la mayoría del recurso polínico proviene de un limitado número de especies y familias de plantas.

Existen reportes de abejas que visitan numerosas plantas pero el recurso polínico proviene de un número restringido de especies (Smith, López, Moreno, Roubik, \& Wcislo, 2015) lo cual coincide con lo observado en este estudio, en el que de los 26 tipos polínicos encontrados en las cargas de T. aethiops dos especies aportan el $91.5 \%$ del recurso polínico total (U. europaeus y $S$. laxum). Ambas especies son introducidas y florecen durante todo el año produciendo abundante polen (Barbosa, Velázquez, \& Mayorga-Saucedo, 2006; Díaz, Díaz, \& Vargas, 2012). Se ha reportado que plantas exóticas que se encuentran en una mayor densidad en áreas alteradas pueden ser más atractivas y proporcionar una mayor recompensa para las abejas, lo que las convierte en el recurso dominante en estas áreas, de modo que su presencia afecta positivamente la comunidad de abejas (Williams, Cariveau, Winfree, \& Kremen, 2011). Además, se ha observado que en áreas urbanas no existe una estricta preferencia por un recurso nativo y las abejas pueden usar principalmente polen de plantas introducidas que se encuentran ampliamente distribuidas (Montalva, Allendes, \& Castro, 2010; MacIvor, Cabral, \& Packer, 2014). El frecuente uso de $U$. europaeus y $S$. laxum demuestra la capacidad de T. aethiops para sobrevivir en ambientes altamente intervenidos, ya que no depende exclusivamente de especies nativas que pueden encontrarse en baja densidad. Además, se observó que las plantas más utilizadas pueden variar mes a mes, lo que muestra la plasticidad que tiene esta abeja en cuanto al uso de recursos polínicos. Esta es una adaptación que puede favorecer su supervivencia en ambientes urbanos.

El polen de plantas como Abatia parviflora (Salicaseae) y Tibouchina lepidota (Melastomataceae) tuvo un aporte total menor pero fueron visitadas frecuentemente por T. aethiops durante algunos meses. Es probable que cuando estas plantas florecen, T. aethiops las visite debido a que se convierten en un recurso abundante y al terminar su fase de floración vuelve a usar plantas como U. europaeus y $S$. laxum, las cuales permanecen en floración durante la mayor parte del año (Barbosa et al., 2006; Díaz et al., 2012). Tipos polínicos que fueron muy escasos durante todos los muestreos pueden pertenecer a plantas que son utilizadas para recolectar néctar (Cane \& Sipes, 2006). En estos casos el polen es adquirido por las abejas de forma indirecta, lo que explica su baja frecuencia de aparición en las cargas de polen.

Gonzáles y Ospina (2008), reportan la presencia de machos de T. aethiops durante todo el año en La Calera, una zona cercana a Bogotá. Nuestros resultados coinciden con lo reportado por dichos autores ya que observamos machos durante todos los muestreos. A pesar de que se registró nidificación constante durante todos los meses, se destacan tres periodos con un mayor número de nidos activos. Estos periodos corresponderían a la fase en que las hembras construyen y aprovisionan los nidos, mientras que los periodos con menos nidos activos comprendería el periodo de desarrollo de la próxima generación de individuos (Roubik, 1992). Los periodos de construcción y aprovisionamiento se observan aproximadamente un mes después de los picos de precipitación. Aunque no hubo correlación entre la precipitación y el número de nidos, es posible pensar que el aumento en el número de nidos obedezca a una mayor disponibilidad de recursos florales causada por los periodos de lluvia previos.

La disminución del número de nidos registrada durante diciembre/2012 y octubre/2013 estuvo influenciada por la instalación de las carpas comerciales, ya que fueron ubicadas 
sobre la entrada de los nidos impidiendo la entrada o salida de las abejas. El efecto de las carpas en diciembre/2013 fue menor que el observado en las ocasiones anteriores, ya que las carpas fueron alejadas de los nidos $50 \mathrm{~cm}$ aproximadamente, de modo que no obstaculizaron la entrada de los nidos. Esto sugiere que el declive en el número de nidos fue causado por factores antrópicos y no hace parte del comportamiento poblacional propio de $T$. aethiops. También, cabe destacar que durante los periodos de disturbios, se observó construcción de nidos en jardines cercanos a aproximadamente $2 \mathrm{~m}$ del agregado de nidos original. Esto muestra la capacidad que tiene esta especie para responder a intervenciones antrópicas.

El periodo de actividad diaria registrado para $T$. aethiops en este estudio concuerda con lo reportado por Vélez y Baquero (2002), quienes observaron que la actividad de forrajeo de T. aethiops sobre Abelia grandiflora (Caprifoliaceae) se presenta entre las 8:00 y las 14:00 h en el Jardín Botánico de Bogotá. En cuanto a la actividad de forrajeo, las entradas a los nidos sin cargas de polen, probablemente corresponda a hembras que forrajeaban por néctar (Roubik, 1992). Durante el primer periodo de muestreos (septiembre-diciembre, 2012) el patrón de actividad indica que las hembras forrajean principalmente por polen en las primeras horas de la mañana, y después de las 11:00 h forrajean para recolectar néctar. El comportamiento de recolección de polen en horas de la mañana se ha reportado tanto para especies de abejas solitarias (González, Mantilla, \& Palacios, 2006) como sociales (Nates-Parra \& Rodríguez, 2011). Después de que se detiene la actividad de forrajeo, cerca de las 14:00 h, es probable que las hembras realicen labores de construcción y mantenimiento de los nidos.

Durante el segundo periodo (enero-abril, 2013) se observó una disminución en el número de entradas de abejas a los nidos lo cual pudo corresponder a con la reducción poblacional a causa de la instalación de las carpas comerciales en el PNEOH. Por otro lado, se observó un cambio en el patrón de actividad de T. aethiops reflejado principalmente en la ausencia del pico de recolección de polen. Esto pudo haber sido causado por una recolonización del área afectada tras el disturbio: posiblemente abejas recién emergidas o llegadas de otras zonas del parque empezaron a construir nuevos nidos, por lo que la recolección de polen se redujo, ya que no tenían celdas para aprovisionar. Sin embargo, el pico de entradas con néctar se mantuvo en un horario similar al del periodo anterior al disturbio, debido a que seguían requiriendo este recurso para su sostenimiento.

Durante el tercer periodo, se registró un número de entradas similar al observado en la primera etapa, debido a que probablemente, después de la reconstrucción de los nidos, las abejas empezaron a aprovisionarlos nuevamente, por lo que su horario de actividad volvió al patrón observado en un principio. Esto coincide con el constante aumento en el número de nidos activos después de los disturbios y sugiere que T. aethiops tiene la capacidad de recuperar su población tras sufrir un evento disruptivo.

El número de entradas a los nidos de $T$. aethiops no varío entre la épocas secas y de lluvia. Esto puede deberse a que las abejas robustas tienen una mayor capacidad de termorregulación (Roberts \& Harrison, 1998) lo que les permite permanecer activas aun cuando las condiciones ambientales no son las óptimas. Estos resultados además coinciden con la falta de correlación entre la temperatura y el número de entradas a los nidos. El menor número de individuos observados durante el periodo seco puede corresponder a una posible reducción en los recursos florales para el aprovisionamiento de nuevos nidos.

Teniendo en cuenta lo expuesto anteriormente, surgen varias consideraciones para tener en cuenta en la conservación de las abejas urbanas. Dearborn y Kark (2009) exponen varias razones por las que se debería incentivar la conservación en áreas urbanas, entre las que destacan que el tener contacto con la biodiversidad permite que las personas se interesen en su protección. También destacan el servicio de polinización que especies de insectos brindan a la creciente agricultura urbana a pequeña escala 
y a las plantas de zonas rurales cercanas de las ciudades (Hunter \& Hunter, 2008).

Fattorini (2011) propone que la conservación en áreas urbanas se debe enfocar en identificar las especies que pueden beneficiarse de los ambientes urbanos, y luego determinar los sitios importantes para su mantenimiento. En este estudio encontramos que T. aethiops tiene la capacidad de utilizar plantas introducidas como recurso polínico, y presumiblemente puede recuperar su población después de sufrir disminuciones importantes causadas por alteraciones antrópicas, propias de ambientes urbanos. Estas son características que probablemente le han permitido sobrevivir en parques y zonas aledañas a Bogotá, por lo que esta especie de abeja sería un candidato ideal para programas de conservación en zonas urbanas.

Por último, es importante destacar la importancia de la información expuesta en el presente estudio, ya que se identifica el recurso polínico utilizado por $T$. aethiops en una zona urbana, lo cual puede ser utilizado en el planteamiento de estrategias de conservación para este polinizador. Del mismo modo, el identificar los picos reproductivos de esta especie permite conocer los periodos en que sería prioritaria la protección de la zona habitada por las abejas con el fin de preservar su población.

\section{AGRADECIMIENTOS}

A Erasmo A. Rodríguez por facilitar los datos de la estación meteorológica del Departamento de Ingeniería Civil y Agrícola de la Universidad Nacional de Colombia. A los integrantes del Laboratorio de Investigación en Abejas de la Universidad Nacional (LABUN) por el apoyo durante la fase de laboratorio. A los evaluadores anónimos por sus aportes. A la Universidad Nacional de Colombia por el apoyo al desarrollo del curso Biología y Comportamiento de Abejas, dentro del cual se enmarcó este trabajo.

\section{RESUMEN}

Thygater aethiops es una abeja nativa que puede encontrarse en parques y jardines en diversas áreas urbanas como aquellas de la ciudad de Bogotá (Colombia). Sin embargo, es poco lo que se conoce sobre su biología y sus adaptaciones ecológicas a áreas urbanas. El presente estudio tuvo como objetivos describir ciclos estacionales y la actividad de forrajeo diaria de T. aethiops, así como identificar el recurso polínico usado por estas abejas a lo largo de un año en una población que nidifica en un agregado en el "Parque Nacional Enrique Olaya Herrera" de Bogotá. Cambios en la actividad de nidificación fueron registrados semanalmente contando el número de nidos activos en el agregado entre diciembre/2012 y febrero/2014. Para determinar el horario de forrajeo diario, se contó el número de abejas que entraban a sus nidos en un periodo de $10 \mathrm{minu}-$ tos cada hora entre las 8:00 y las 14:00 h. Semanalmente se capturaron hembras que estuvieran regresando a sus nidos con cargas de polen, entre septiembre/2012 y agosto/2013; dichas cargas fueron analizadas. Se observó tres picos de nidificación tras los picos de precipitación, pero el número de nidos activos no estuvo correlacionado con la precipitación. El número de nidos activos se redujo (20-50 \% de los nidos) después de que se registrara un disturbio antrópico en el área de nidificación. Las abejas forrajean por polen y néctar entre las 8:00 y 14:00 h con un pico de actividad a las 10:00 h. La actividad de forrajeo diaria cambió durante el periodo de estudio debido a disturbios antrópicos. No hubo una relación significativa entre la temperatura del aire y el número de entradas a los nidos. El horario de actividad no cambió entre los periodos secos y lluviosos. Se encontró un total de 26 tipos polínicos en 169 cargas de polen. Ulex europaeus (Fabaceae) y Solanum laxum (Solanaceae) fueron las plantas más abundantes representadas en las cargas de polen durante todo el periodo de estudio. De acuerdo a estos resultados, T. aethios sería considerada una especie mesoléctica. La habilidad de T. aethiops para utilizar diferentes recursos polínicos tanto nativos como introducidos, así como su presumible capacidad para recuperar su población después de disturbios son características que le pueden haber permitido adaptarse a ambientes urbanos. El conocimiento de los recursos florales, así como otras características biológicas de esta abeja son importantes para promover su conservación en áreas urbanas.

Palabras clave: abejas urbanas, conservación, Eucerini, Solanum laxum, Ulex europaeus.

\section{REFERENCIAS}

Barbosa, L., Velázquez, A., \& Mayorga-Saucedo, R. (2006). Solanaceae composition, pollination and seed dispersal syndromes in Mexican Mountain Cloud Forest. Acta Botanica Brasilica, 20(3), 599-613.

Cane, J. H., \& Sipes, S. 2006. Characterizing floral specialization by bees: analytical methods and a revised lexicon for oligolecty. In N. M. Waser, \& J. Ollerton (Eds.), Plant-Pollinator Interactions from specialization to generalization (pp. 99-122). Chicago: The University of Chicago Press. 
Da Silveira, F. A. (1991). Influence of pollen grain volume on the estimation of the relative importance of its source to bees. Apidologie, 22, 495-502.

Departamento Administrativo Nacional de Estadística (DANE). (2014). Recuperado de https://www.dane. gov.co/index.php/poblacion-y-demografia/censos

Dalmazzo, M. (2010). Diversidad y aspectos biológicos de abejas silvestres de un ambiente urbano y otro natural de la región central de Santa Fe, Argentina. Revista de la Sociedad Entomológica Argentina, 69(1-2), 33-44.

Dearborn, D., \& Kark, S. (2009). Motivations for conserving urban biodiversity. Conservation Biology, 24(2), 432-440.

Díaz, A. M., Díaz, J. E., \& Vargas, O. (Eds.). (2012). Catálogo de plantas invasoras de los humedales de Bogotá. Bogotá: Grupo de Restauración Ecológica de la Universidad Nacional de Colombia y Secretaría Distrital de Ambiente.

Fattorini, S. (2011). Insect extinction by urbanization: A long-term study in Rome. Biological Conservation, 144(1), 370-375

Fernández-Alonso, J. L. (2008). Estudio en Labiatae - VI. Hibridación en el género Salvia en Colombia y su interés horticultural. Caldasia, 30(1), 21-48.

Fonnegra, R. J. (1989). Métodos de estudio palinológico. Medellín: Centro de Investigaciones Facultad de Ciencias Exactas y Naturales, Universidad de Antioquia.

González, V., Mantilla, B., \& Palacios, E. (2006). Foraging activity of the solitary andean bee, Anthophora walteri (Hymenoptera: Apidae, Anthophorini). Revista Colombiana de Entomología, 32(1), 73-76.

González, V. H., \& Ospina, M. (2008). Nest Structure, seasonality, and host plants of Thygater aethiops (Hymenoptera: Apidae, Eucerini) in the Andes. Journal of Hymenoptera Research, 17(1), 110-115.

González, V. H., Ospina, M., \& Bennet, D. J. (2005). Abejas altoandinas de Colombia. Bogotá: Instituto de Investigación de Recursos Biológicos Alexander Von Humboldt.

González, V. H., Velez-Ruiz, R. I., \& Engel, M. S. (2014). A new paracolletine bee from Colombia (Hymenoptera: Colletidae), with an updated checklist of the tropical Andean bee fauna. Journal of Melittology, 43, 1-26.

Hernández, J. L., Frankie, G. W., \& Thorp, R. W. (2009). Ecology of urban bees: a review of current knowledge and directions for future study. Cities and the Environment, 2(1), 1-15.

Hunter, R. \& Hunter, M. D. (2008). Designing for conservation of insects in the built environment. Insect Conservation and diversity, 1(4), 189-196.
Instituto de Hidrología, Meteorología y Estudios Ambientales (IDEAM). (2014). Recuperado de http://institucional.ideam.gov.co/jsp/clima 49

Instituto Distrital de Recreación y Deporte (IDRD). (2014). Recuperado de http://www.idrd.gov.co/web/ htms/seccion-parque-nacional-enrique-olaya-herrera_1306.html

Kratochwil, A. (2003) Bees (Hymenoptera: Apoidea) as key-stone species: specifics of resources and requisite utilization in different habitat types. Berichte der Reinhold-Tüxen-Gesellschaft, 15(1), 59-77.

Louveaux, J., Maurizio, A., \& Vorwohl, G. (1978). Methods of Melissopalynology. Bee World, 59(1), 139-157.

Mcintyre, N. E., \& Hostetler, M. E. (2001). Effects of urban land use on pollinator (Hymenoptera: Apoidea) communities in a desert metropolis. Basic and Applied Ecology, 2(1), 209-218.

MacIvor, J. S., Cabral, J. M., \& Packer, L. (2014). Pollen specialization by solitary bees in an urban landscape. Urban Ecosystems, 17(1), 139-147.

Montalva, J., Allendes, J. L., \& Castro B. (2010). Las abejas (Hymenoptera:Apoidea) del Jardín Botánico Chagual. Estudio de caso de abejas nativas en zonas urbanas de Santiago de Chile. Revista Chagual, 8(1), 13-23.

Nates-Parra, G. \& Rodríguez, A. (2011). Forrajeo en colonias de Melipona eburnea (Hymenoptera: Apidae) en el piedemonte llanero (Meta, Colombia). Revista Colombiana de Entomología, 37(1), 121-127.

Nates-Parra, G., Parra, A., Rodríguez, A., Baquero, P., \& Vélez, E. (2006). Abejas silvestres (Hymenoptera: Apoidea) en ecosistemas urbanos: Estudio en la ciudad de Bogotá y sus alrededores. Revista Colombiana de Entomología, 32(1), 77-84.

Pellkofer, S. D. (2011). The effects of local and landscape-level characteristics on the abundance and diversity of solitary-nesting Hymenoptera in urban family gardens (Master's thesis). University of Zurich, Zurich, Suiza.

Pinilla-Gallego, M. S., \& Nates-Parra, G. (2015). Diversidad de visitantes y aproximación al uso de nidos trampa para Xylocopa (Hymenoptera: Apidae) en una zona productora de pasifloras en Colombia. Actualidades Biológicas, 37(103), 143-153. doi: 10.17533/ udea.acbi.v37n103a03

Quijano, C., Parra, A., \& Nates-Parra, G. (2002). Observaciones preliminares de la estructura y arquitectura de los nidos de Thygater aethiops mediante el uso de moldes de parafina. Resumen I Encuentro Colombiano Sobre Abejas Silvestres, Bogotá, Colombia. 
R Core Team, A Language and Environment for Statistical. (2014). R Foundation for Statistical Computing. Retrieved from http://www.r-project.org

Roberts, S., \& Harrison, J. (1998). Mechanisms of thermoregulation in flying bees. American Zoologist, $38(1), 492-502$.

Roubik, D. W. (1992). Ecology and natural history of tropical bees. Cambridge: Cambridge University Press.

Sipes, S. D., \& Tepedino, V. J. (2005). Pollen-host specificity and evolutionary patterns of host switching in a clade of specialist bees (Apoidea: Diadasia). Biological Journal of the Linnean Society, 86(4), 487-505.

Smith, A. R., López, I. J., Moreno, J. E., Roubik, D. W., \& Wcislo, W. T. (2012). Pollen use by Megalopta sweat bees in relation to resource availability in a tropical forest. Ecological Entomology, 37(4), 309-317.

Vaissiere, B., Freitas, B. M., \& Gemmill-Herren, B. (2011). Protocol to detect and assess pollination deficits in crops: a handbook for its use. Roma: Food and agriculture organization of the United Nations (FAO).

Vélez, E. D. \& Baquero, P. (2002). Observaciones preliminares del comportamiento de forrajeo de Thygater aethiops sobre Abelia grandiflora en el jardín botánico "José Celestino Mutis". Resumen I Encuentro Colombiano Sobre Abejas Silvestres, Bogotá, Colombia.

Williams, N. M., Cariveau, D., Winfree, R., \& Kremen, C. (2011). Bees in disturbed habitats use, but not prefer, alien plants. Basic and Applied Ecology, 12(1), 332-341.

\section{Ver Apéndice Digital / See Digital Appendix}


